\title{
Usual interstitial pneumonia in rheumatoid arthritis-associated interstitial lung disease
}

\author{
E.J. Kim*, B.M. Elicker" , F. Maldonado", W.R. Webb”, J.H. Ryu^, J.H. Van Uden\#, \\ J.S. Lee*, T.E. King Jr* and H.R. Collard*
}

ABSTRACT: Interstitial lung disease is a common manifestation of rheumatoid arthritis; however, little is known about factors that influence its prognosis. The aim of the present study was to determine whether or not the usual interstitial pneumonia pattern found on high-resolution computed tomography (HRCT) is of prognostic significance in rheumatoid arthritis-associated interstitial lung disease (RA-ILD).

Patients with RA-ILD were identified retrospectively $(n=82)$. The relationship of a definite usual interstitial pneumonia pattern on HRCT to survival was determined and compared to that in a cohort of patients with radiologically diagnosed idiopathic pulmonary fibrosis $(n=51)$.

A definite usual interstitial pneumonia pattern was seen in 20 (24\%) out of 82 patients with RAILD. These patients showed worse survival than those without this pattern (median survival 3.2 versus $6.6 \mathrm{yrs}$ ), and a similar survival to those with idiopathic pulmonary fibrosis. On multivariate analysis, a definite usual interstitial pneumonia pattern on HRCT was associated with worse survival (hazard ratio of 2.3). Analysis of specific HRCT features demonstrated that traction bronchiectasis and honeycomb fibrosis were associated with worse survival (hazard ratio of 2.6 and 2.1, respectively). Female sex (hazard ratio of 0.30 ) and a higher baseline diffusing capacity of the lung for carbon monoxide (hazard ratio of 0.96 ) were associated with better survival.

A definite usual interstitial pneumonia pattern on HRCT has important prognostic implications in RA-ILD.

KEYWORDS: Computed tomography, interstitial lung diseases, prognosis, rheumatoid arthritis nterstitial lung disease (ILD) is a common extra-articular manifestation of rheumatoid arthritis (RA), and a significant cause of morbidity and mortality in this patient population [1]. Using high-resolution computed tomography (HRCT) scanning, the estimated prevalence of RAassociated ILD (RA-ILD) among patients with RA is $19-56 \%$ [2-5]. Despite this considerable prevalence, little is known about the natural history of RA-ILD or features associated with prognosis.

Among patients with idiopathic interstitial pneumonia (IIP), HRCT and histopathological patterns have been shown to be of important prognostic significance [6, 7]. Most notably, the usual interstitial pneumonia (UIP) pattern, the histopathological and radiological pattern found in patients with idiopathic pulmonary fibrosis (IPF), is associated with poorer outcomes [7-9]. It remains unknown whether the UIP pattern has the same prognostic implications for patients with RA-ILD as it does for those with IPF.
Unlike other forms of connective tissue diseaserelated ILD (e.g. scleroderma, polymyositis, dermatomyositis, Sjögren's syndrome and undifferentiated connective tissue disease), in which the nonspecific interstitial pneumonia (NSIP) pattern predominates [10-18], RA-ILD has a higher proportion of patients with the UIP pattern [19, 20]. Patients with RA-ILD infrequently undergo surgical lung biopsy, making comparative histopathological studies in RA-ILD challenging and at risk of selection bias.

Data from the IIP population have demonstrated that HRCT can accurately predict the presence of a histopathological UIP pattern in a subset of patients [7, 21, 22], and several studies in RA-ILD have suggested a similar specificity $[19,20,23$, 24]. HRCT is noninvasive and commonly performed in RA-ILD patients, making it an ideal method for evaluating the prognostic significance of the underlying pattern. It was hypothesised that a definite UIP pattern on HRCT in patients
AFFILIATIONS

*Depts of Medicine,

\# Radiology, University of California, San Francisco, CA, and "Dept of Medicine, Division of Pulmonary and Critical Care Medicine, Mayo Clinic, Rochester, MN, USA.

CORRESPONDENCE

E.J. Kim

505 Parnassus Avenue

Campus Box 0111

San Francisco

CA 94143

USA

E-mail: ekim@ucsf.edu

Received:

June 102009

Accepted after revision:

Nov 262009

First published online:

Dec 082009 
with RA (RA-UIP) would predict worse survival than non-UIP patterns (RA-non-UIP), and that the survival of patients with RA-UIP would mirror that found in patients with IPF.

\section{MATERIALS AND METHODS Study population}

Patients with RA-ILD and IPF were identified from the longitudinal cohorts of ILD patients seen at the University of California, San Francisco (UCSF; CA, USA) and the Mayo Clinic (Rochester, MN, USA) between January 1, 2001 and November 1, 2008. Enrolment into these cohorts included permission to review all medical records. Demographics, clinical features, medication history and pulmonary function test results were obtained for all patients via medical record chart review. The Institutional Human Subject Review Committee at each institution approved the protocol.

Rheumatoid arthritis-associated ILD

Patients with RA at UCSF and the Mayo Clinic were included in the present study if they had: 1) a diagnosis of RA based on American College of Rheumatology criteria [25];2) evidence of diffuse ILD on HRCT (as defined by the interpreting radiologist); and 3) undergone HRCT within $1 \mathrm{yr}$ of ILD diagnosis that was available for review. Medical records were screened to exclude other likely causes of ILD.

IPF

A retrospective cohort of patients with radiologically diagnosed IPF seen at UCSF was used as a comparison group. Radiologically diagnosed IPF was defined by the presence of a definite UIP pattern on HRCT in patients who met modified American Thoracic Society (ATS)/European Respiratory Society (ERS) consensus diagnostic criteria [6].

\section{HRCT analysis}

HRCT scans from subjects with RA-ILD were reviewed independently in a blinded fashion by two thoracic radiologists experienced in the interpretation of diffuse lung disease, with disagreements resolved by consensus. Standard highresolution protocols were used to obtain images for evaluation. As this was a retrospective study, there was no single protocol that was utilised for all patients. All images were $<2.0 \mathrm{~mm}$ in collimation and were reconstructed using high-resolution algorithms. In most cases, supine, prone and expiratory images were available for review. Each scan was scored as a definite UIP or not a definite UIP pattern, with the latter group further separated into indeterminate UIP/NSIP, likely NSIP and other. Determination of a definite UIP pattern was based on previously published guidelines [6]. Briefly, scans consistent with definite UIP contained basilar predominant reticulation, traction bronchiectasis and honeycombing, with limited ground-glass abnormality. Predominant bibasilar ground-glass attenuation with limited-to-no reticulation and absent honeycombing was consistent with likely NSIP. Ground-glass attenuation, consolidation, reticulation, traction bronchiectasis and honeycombing were scored as absent, mild or moderate/ severe for each HRCT scan.

\section{Histopathological analysis}

All available lung biopsy specimens were reviewed prospectively at the time of initial enrolment in the parent longitudinal cohort studies by a lung pathologist at each centre with experience in the evaluation of diffuse lung disease, and were classified using the histopathological patterns described in the ATS/ERS international consensus classification of the IIPs [6]

\section{Statistical analysis}

The primary analysis compared RA-UIP (as defined by the presence of a definite UIP pattern on HRCT) with RA-non-UIP (as defined by the absence of a definite UIP pattern on HRCT). Both groups were also compared with radiologically diagnosed IPF. Descriptive data are presented as mean \pm SD unless otherwise indicated. Intergroup comparisons were performed using an unpaired t-test or the Wilcoxon rank-sum test as appropriate for continuous variables, and the Chi-squared test or Fisher's exact test as appropriate for categorical variables. Survival time was defined as time from initial clinic visit to death (as determined by review of clinic records and the Social Security Death Index) or censoring. Kaplan-Meier curves were generated and compared using the log-rank test. Cox's regression analysis was performed in order to assess the predictive value of HRCT pattern and selected covariates identified a priori on survival time. Any variable with a p-value of $<0.1$ on bivariate analysis was included in multivariate analysis. All statistical analyses were performed using SAS version 9.1 (SAS Institute, Cary, NC, USA). Significance was defined as a $p$-value of $\leqslant 0.05$.

\section{RESULTS}

\section{Patient population}

Patients with RA-ILD were identified ( $\mathrm{n}=99)$, of whom 84 had HRCT scans that were available for review and performed within $1 \mathrm{yr}$ of the initial clinic visit (fig. 1). Two of these patients had HRCT patterns categorised as other (one organising pneumonia and one bronchiolitis), and were excluded from additional analyses as they did not have diffuse disease. Of the remaining 82 patients, an HRCT pattern of

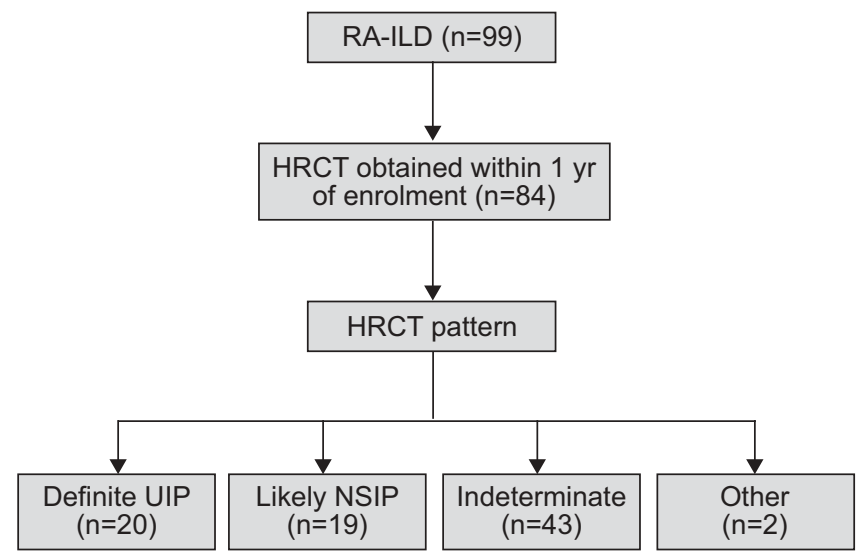

FIGURE 1. Flow chart showing the high-resolution computed tomography (HRCT) pattern in patients with rheumatoid arthritis-associated interstitial lung disease (RA-ILD). Of 99 patients with RA-ILD, 84 had HRCT scans available within $1 \mathrm{yr}$ of initial presentation and enrolment into a longitudinal cohort. The HRCT pattern distribution is shown. The two cases with an alternative pattern (organising pneumonia $(n=1)$ and bronchiolitis $(n=1))$ were excluded from the study analysis. NSIP: nonspecific interstitial pneumonia; UIP: usual interstitial pneumonia 
definite UIP was present in 20 (24\%; $\kappa$ 0.54), likely NSIP in 19 $(23 \%)$ and indeterminate in 43 (52\%).

Patient demographics and the clinical characteristics of the 82 RA-ILD patients are presented in table 1 . Comparing the RAUIP, RA-non-UIP and IPF groups, only age and sex differed significantly. When compared directly, there were no significant differences in age, sex or pulmonary function between the RA-UIP group and either the RA-non-UIP or IPF group. The RA-UIP group had fewer smokers than the RA-non-UIP group $(p=0.05)$. Longer RA disease duration was present in the RAUIP group $(p=0.03)$. There were no significant differences in demographics or baseline characteristics between patients with RA-ILD and likely NSIP and those with an indeterminate UIP/ NSIP pattern on HRCT (data not shown).

\section{RA-UIP and survival time}

The overall median duration of follow-up in the RA-ILD cohort was 3.9 yrs (range 0.3-7.5 yrs). Median follow-up in the RAUIP group was 3.9 yrs (0.5-7.5 yrs) and, in the RA-non-UIP group, 3.9 yrs (0.3-7.5 yrs). Cause of death data were available for only a small number of the RA-ILD cohort. Of the eight known causes of death, seven were pulmonary in nature and were felt to be at least partially related to the underlying RAILD.

The median survival time for all RA-ILD subjects was 5.0 yrs. RA-UIP had a worse median survival time than RA-non-UIP (3.2 versus $6.6 \mathrm{yrs}$, respectively; $\mathrm{p}=0.04$ ) (fig. 2 ). Median survival time did not differ between the RA-UIP and IPF groups $(p=0.66)$. On bivariate analysis, sex, baseline percentage predicted forced vital capacity (FVC), baseline percentage predicted diffusing capacity of the lung for carbon monoxide (DL,CO) and a definite UIP pattern on HRCT were associated with survival time in RA-ILD (table 2). Age, duration of RA and history of smoking were not predictive. Cox's regression

\begin{tabular}{|c|c|c|c|c|c|c|}
\hline \multirow[t]{3}{*}{ TABLE 1} & \multicolumn{6}{|c|}{$\begin{array}{l}\text { mographic and clinical characteristics of } \\
\text { umatoid arthritis-associated interstitial lung } \\
\text { ease patients }\end{array}$} \\
\hline & \multicolumn{3}{|c|}{ Population } & \multicolumn{3}{|c|}{ p-value } \\
\hline & RA-UIP & $\begin{array}{l}\text { RA-non- } \\
\text { UIP }\end{array}$ & IPF & Overall & $\begin{array}{c}\text { RA-non- } \\
\text { UIP\# }\end{array}$ & $\mathrm{IPF}^{\#}$ \\
\hline Subjects $n$ & 20 & 62 & 51 & & & \\
\hline Age yrs & $69 \pm 6$ & $65 \pm 10$ & $72 \pm 9$ & 0.001 & 0.15 & 0.08 \\
\hline Male sex & 55 & 50 & 75 & 0.03 & 0.70 & 0.11 \\
\hline Duration of RA yrs & $7(3-30)$ & $2(0-9)$ & NA & NA & 0.03 & NA \\
\hline Ever-smoker & 55 & 77 & 76 & 0.12 & 0.05 & 0.07 \\
\hline FVC \% pred & $66 \pm 25$ & $70 \pm 20$ & $69 \pm 21$ & 0.72 & 0.39 & 0.63 \\
\hline DL,Co \% pred & $50 \pm 22$ & $48 \pm 21$ & $41 \pm 16$ & 0.10 & 0.56 & 0.07 \\
\hline
\end{tabular}

Data are presented as mean $\pm \mathrm{SD}$, percentage or median (interquartile range), unless otherwise indicated. RA-UIP: usual interstitial pneumonia (UIP) pattern in patients with rheumatoid arthritis (RA); RA-non-UIP: non-UIP pattern in patients with RA; IPF: idiopathic pulmonary fibrosis; FVC: forced vital capacity; DL,CO: diffusing capacity of the lung for carbon monoxide; NA: not applicable; \% pred: percentage of the predicted value. ${ }^{\#}$ : versus RA-UIP. modelling demonstrated that a definite UIP pattern on HRCT, female sex and increased baseline $D \mathrm{~L}, \mathrm{CO}$ were associated with survival time (table 2).

\section{HRCT pattern, radiological features and survival time}

Estimated survival times were compared between RA-ILD patients with a definite UIP pattern, a likely NSIP pattern and an indeterminate UIP/NSIP pattern on HRCT (fig. 3). RA-ILD patients with a definite UIP pattern showed worse survival than those with a likely NSIP pattern $(p=0.02)$. Survival in patients with an indeterminate pattern did not differ from those with a definite UIP pattern or a likely NSIP pattern $(p=0.15$ and $p=0.19$, respectively). On bivariate survival analysis of specific HRCT features in RA-ILD patients, reticulation, traction bronchiectasis and honeycombing were significantly associated with worse survival time. Cox's regression modelling found the presence and extent of traction bronchiectasis and honeycombing to be significant independent predictors of worse survival time (hazard ratio of 2.6 $(\mathrm{p}=0.02)$ and HR $2.1(\mathrm{p}=0.002)$, respectively).

\section{HRCT pattern and histopathology}

Eighteen (22\%) of the RA-ILD patients had histopathological data available; four were from transbronchial biopsy specimens, one from an explanted lung and the remaining 13 from surgical lung biopsy specimens (fig. 4). The median time from initial HRCT to obtaining a tissue specimen was 80 days (range $-903-3,606$ days). In the majority of the patients $(72 \%)$, the tissue was obtained within $1 \mathrm{yr}$ of the initial HRCT.

Only one (5\%) out of 20 patients with a definite UIP pattern on HRCT underwent surgical lung biopsy. This patient, who underwent surgical lung biopsy in order to help diagnose a focal pulmonary process and not the underlying chronic lung disease, had necrotising pneumonia without note of an underlying ILD. Another patient with a definite UIP pattern

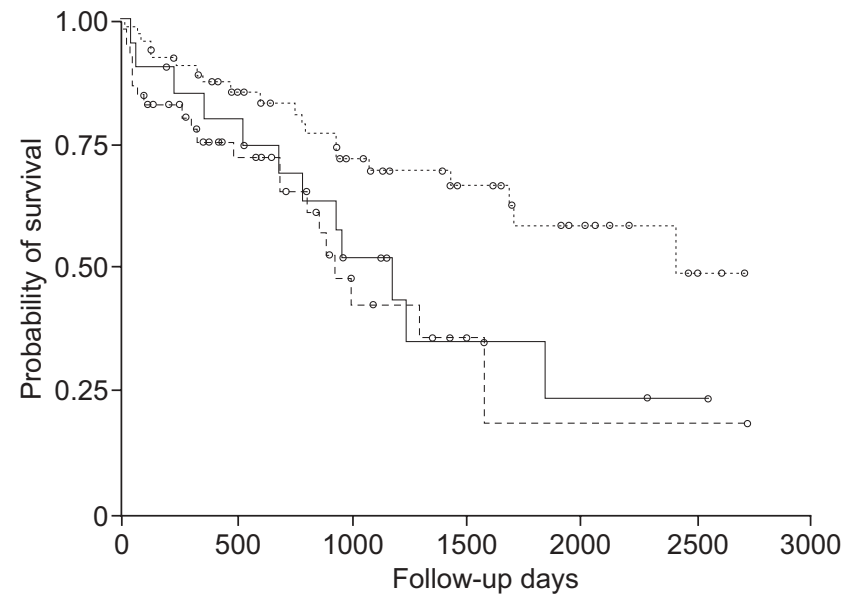

FIGURE 2. Kaplan-Meier survival curve for patients with a rheumatoid arthritis (RA)-associated usual interstitial pneumonia (UIP) pattern (RA-UIP; - - a RAassociated non-UIP pattern (RA-non-UIP; .....) and idiopathic pulmonary fibrosis (IPF; ------). Comparing across all three groups, there were significant differences in survival estimates $(p=0.01)$. The estimated median survival in RA-UIP was significantly worse than that in RA-non-UIP (3.2 versus $6.6 \mathrm{yrs} ; \mathrm{p}=0.04$ ) Estimated median survival in RA-UIP did not differ significantly from that in IPF (3.2 versus 2.6 yrs; $p=0.66$ ). 


\begin{tabular}{|c|c|c|c|c|c|}
\hline \multirow[t]{3}{*}{ TABLE 2} & \multicolumn{5}{|c|}{$\begin{array}{l}\text { Predictors of survival time in patients with } \\
\text { rheumatoid arthritis-associated interstitial lung } \\
\text { disease }\end{array}$} \\
\hline & & \multicolumn{2}{|c|}{ Bivariate } & \multicolumn{2}{|c|}{ Multivariate } \\
\hline & & Hazard ratio & $\mathrm{p}$-value & Hazard ratio & $p$-value \\
\hline \multicolumn{2}{|l|}{ Female sex } & 0.55 & 0.10 & 0.30 & 0.008 \\
\hline \multicolumn{2}{|c|}{ Baseline FVC (\% pred) } & 0.98 & 0.01 & & \\
\hline \multicolumn{2}{|c|}{ Baseline DL,Co (\% pred) } & 0.97 & 0.002 & 0.96 & 0.003 \\
\hline \multicolumn{2}{|c|}{ Definite UIP pattern" } & 2.09 & 0.04 & 2.34 & 0.05 \\
\hline \multicolumn{6}{|c|}{$\begin{array}{l}\text { Hazard ratios reflect the relative risk of death associated with the given variable } \\
\text { (or, for continuous variables, a one-unit increase in the variable). FVC: forced } \\
\text { vital capacity; } D \text { L,CO: diffusing capacity of the lung for carbon monoxide; UIP: } \\
\text { usual interstitial pneumonia; \% pred: percentage of the predicted value. }{ }^{\# \text { : on }} \\
\text { high-resolution computed tomography. }\end{array}$} \\
\hline
\end{tabular}

on HRCT underwent transbronchial biopsy procedures that were indeterminate. Of the 19 patients with a likely NSIP pattern on HRCT, six (32\%) underwent surgical lung biopsy (final histopathology: four UIP, and two NSIP), two underwent transbronchial biopsy (final histopathology: one organising pneumonia, and one indeterminate) and one had an explanted lung reviewed (final histopathology: UIP). Finally, seven (16\%) out of 43 patients with an indeterminate pattern on HRCT underwent surgical lung biopsy (final histopathology: five UIP, and one NSIP) and one underwent transbronchial biopsy (final histopathology: diffuse alveolar damage).

\section{Treatment regimen and survival in RA-ILD}

The medication regimens at the initial clinical visit and last follow-up were reviewed (fig. 5). The majority (80\%) of patients were treated with a regimen that included more than one agent. Prednisone was the most common (71\%) medication used. Methotrexate and an anti-tumour necrosis factor (antiTNF) agent were the next most commonly (28 and $27 \%$, respectively) used. Methotrexate and anti-TNF therapies were not associated with worse survival (data not shown).

\section{DISCUSSION}

The results of the present study demonstrate the prognostic significance of an HRCT pattern of definite UIP in patients with RA-ILD. As is true for patients with IPF compared with patients with other IIPs, patients with RA-UIP exhibit worse survival than patients with RA-non-UIP. Indeed, there was no detectable difference in survival between patients with RA-UIP and those with IPF in the present study. In further support of these findings, the extent of traction bronchiectasis and honeycomb fibrosis on HRCT were both powerful radiological predictors of survival time in RA-ILD. These data suggest that the identification of the UIP pattern in patients with RA-ILD is clinically relevant, both in defining the stage of disease (RAUIP with minimal honeycomb fibrosis suggesting earlier disease) and the overall prognosis of the patient with RA-UIP. Similar relationships between the extent of honeycomb fibrosis and survival have been found in patients with IPF [26, 27].

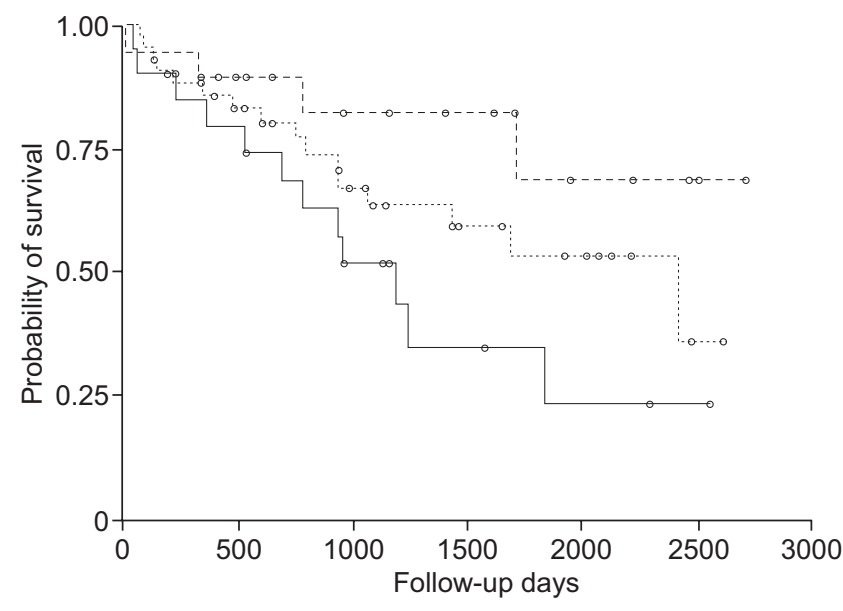

FIGURE 3. Kaplan-Meier survival curve for patients with rheumatoid arthritisassociated interstitial lung disease based on high-resolution computed tomography (HRCT) pattern. Comparing the three main HRCT patterns (_ $\_$: definite usual interstitial pneumonia (UIP); ------: likely nonspecific interstitial pneumonia (NSIP); ......: indeterminate UIP/NSIP), there was a trend toward differences in survival estimates $(p=0.06)$. The estimated median survival in definite UIP was significantly worse than that in likely NSIP (3.2 yrs versus incalculable; $p=0.02$ ). The estimated median survival for an indeterminate UIP/NSIP pattern did not differ from that for definite UIP and likely NSIP (6.6 versus 3.2 yrs $(p=0.15)$ and 6.6 yrs versus incalculable $(p=0.19)$, respectively).

\section{UIP pattern and survival}

Several studies of patients with RA-ILD have suggested a relationship of the UIP pattern to survival [20, 28-30]. YOUSEM et al. [28] described 40 patients with RA-ILD, five of whom had a UIP pattern on surgical lung biopsy. Four of the UIP-pattern patients died during follow-up, whereas only one of the nonUIP-pattern patients died. HAKALA et al. [29] reported on 24 patients with RA-ILD. Two patients died; both had honeycombing present on surgical biopsy. YOsHINOUCHI et al. [20] studied 16 patients with RA-ILD; nine had a UIP pattern and seven an NSIP pattern. Contrary to other studies, survival in the NSIP group was worse (57 versus $67 \%$ ), although two of the three NSIP deaths were from nonrespiratory causes. Park et al. [30] reported on 28 biopsy-proven RA-ILD patients as part of a larger connective tissue disease-related ILD cohort. This study found a trend towards worse survival in RA-UIP patients than in RA-ILD patients with an NSIP pattern (hazard ratio 18.95 (95\% confidence interval $0.67-513.65) ; \mathrm{p}=0.08$ ). These data support the present findings.

The present data show a longer duration of RA in the RA-UIP cohort, suggesting that RA-non-UIP may progress to RA-UIP over time. There are limited data addressing this hypothesis in patients with RA-ILD, and they are discordant. One study of RA-ILD patients found that those with a predominant reticular pattern on HRCT had a longer duration of RA than those with a predominant ground-glass pattern [31]. However, a subsequent study of RA-ILD found that patients with an HRCT pattern consistent with NSIP had a longer duration of articular symptoms than did those with an HRCT pattern consistent with UIP [5]. It remains unclear whether RA-non-UIP and RA-UIP represent a continuum of disease or are separate entities. 


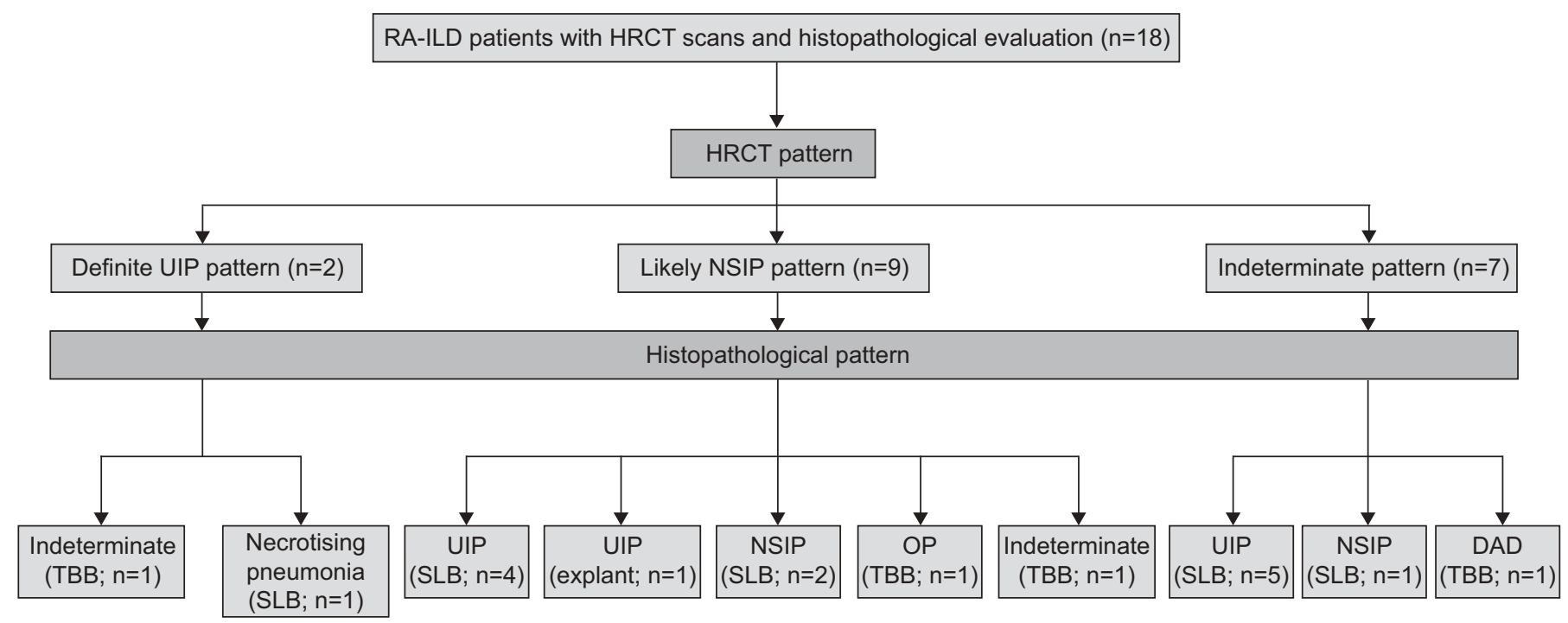

FIGURE 4. Flow chart of histopathology and high-resolution computed tomography (HRCT) pattern in patients with rheumatoid arthritis-associated interstitial lung disease (RA-ILD). Histopathology was available for 18 of the RA-ILD patients. UIP: usual interstitial pneumonia; NSIP: nonspecific interstitial pneumonia; TBB: transbronchial biopsy; SLB: surgical lung biopsy; OP: organising pneumonia; DAD: diffuse alveolar damage.

\section{Relationship of HRCT pattern and histopathology}

Patients with RA-ILD infrequently undergo surgical lung biopsy, making comparative histopathological studies in RAILD challenging and at risk of selection bias. Few of the present RA-ILD patients underwent surgical lung biopsy; only two patients with definite UIP on HRCT underwent histopathological evaluation (one surgical lung biopsy and one transbronchial biopsy). Thus it is not possible to comment on the correlation of a definite UIP pattern on HRCT and histopathology. However,

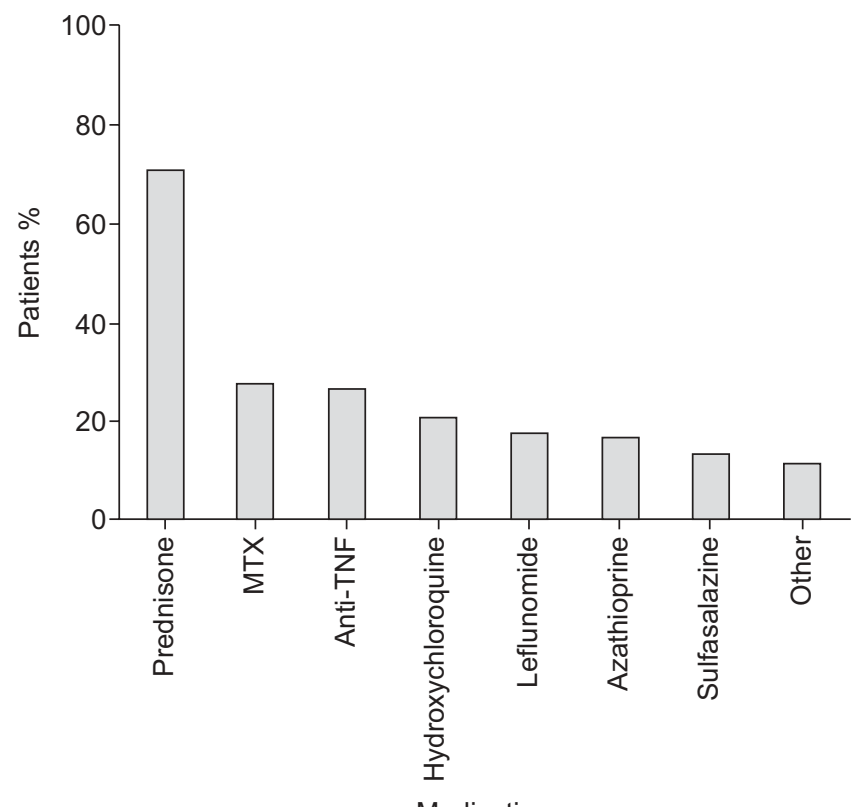

FIGURE 5. Medication use in rheumatoid arthritis-associated interstitial lung disease patients. Medication regimens from the time of the initial and last follow-up visit were reviewed. Prednisone was the most common agent prescribed, followed by methotrexate (MTX) and an anti-tumour necrosis factor (anti-TNF) agent. studies in both RA-ILD and IIP have demonstrated that a definite UIP pattern on HRCT is highly specific for UIP on biopsy [19, 21].

Tissue examination was more often sought in patients with a non-UIP HRCT pattern. The present data show that, in the absence of a definite UIP radiographic pattern, the histopathological findings are difficult to predict. In cases with a radiological NSIP pattern or indeterminate pattern, a UIP pattern is commonly found on histopathological examination. These results mirror findings in studies of IIP patients [7].

\section{Impact of treatment on survival}

No relationship was found between the use of anti-TNF agents and survival time in the present cohort of RA-ILD patients. There have been several case reports of anti-TNF agent use and acute worsening or death in RA-ILD patients [32-34]. However, a retrospective study utilising a national registry of $>17,000$ RA patients did not find any association between current anti-TNF use (infliximab or etanercept) and hospitalisation related to ILD [35].

There are several limitations to the present study. First, it is a retrospective study based on patients seen at pulmonary clinics at two tertiary care referral centres. It is likely that the patients seen at the centres represent a population with more advanced or difficult-to-treat disease, thereby introducing a selection bias. Compared to RA-ILD subjects in other recently published studies $[19,30]$, the present patients had a similar percentage predicted FVC (69 versus $67 \%$ in prior studies), but a somewhat lower DL,CO at baseline (48 versus $53-63 \%$ in prior studies).

Secondly, it is recognised that some histopathological UIP has been misclassified as non-UIP (indeed, this is why only radiologically diagnosed IPF patients were included in the control group). Given the high degree of specificity of a definite UIP pattern on HRCT for histopathological UIP, it is 
unlikely that definite UIP cases were misclassified. Thus the discordance between radiological and histopathological pattern has, if anything, biased the results toward the null hypothesis, by reducing the mean survival of the non-UIP group.

Finally, we are aware that there are other factors that could have influenced mortality in the RA-ILD group. It was not possible to obtain information on extra-articular manifestations of RA (which are known to increase mortality) in the present RA-ILD cohort. We are not aware of any data that suggest that there is a difference in the prevalence of extra-articular manifestations based on ILD pattern (i.e. RA-UIP versus RAnon-UIP), or that nonpulmonary deaths are preferentially increased in either group, but these issues should be addressed in subsequent studies.

In conclusion, the present study demonstrates that a UIP pattern on HRCT is clinically relevant, and suggests that all RA-ILD patients should undergo evaluation of their HRCT pattern. HRCT is widely available, reliable in the hands of experienced radiologists, low cost and low risk compared to surgical lung biopsy. Quantification of the extent of radiological fibrosis lends additional prognostic value. Patients with a UIP pattern and extensive fibrosis on HRCT should be counselled regarding their poor prognosis, and appropriate patients should be considered for lung transplantation. Whether or not RA-UIP is less responsive to immunomodulatory therapy than RA-non-UIP is unknown, and further studies are required in order to answer this question. In patients with an indeterminate or likely NSIP pattern on $\mathrm{HRCT}$, it remains unresolved whether or not surgical lung biopsy should be pursued. It is clear that many such cases would demonstrate histopathological UIP, similar to what is seen in the IIPs. The clinical implications of these radiologically atypical UIP cases should be the focus of future research.

\section{SUPPORT STATEMENT}

This study was funded by National Heart, Lung, and Blood Institute (Bethesda, MD, USA) grant number HL086516.

\section{STATEMENT OF INTEREST}

Statements of interest for H.R. Collard and T.E. King Jr can be found at www.erj.ersjournals.com $/ \mathrm{misc} /$ statements.dtl

\section{ACKNOWLEDGEMENTS}

We would like to thank J. Berkeley, D. Koehler and S. McLaughlin (all University of California, San Francisco, CA, USA) for their assistance in identifying subjects for the present study, and their work in maintaining the interstitial lung disease (ILD) database. In addition, we would like to express our gratitude to the ILD patients who participate in our database, as well as to the providers of the University of California, San Francisco, ILD Consortium.

\section{REFERENCES}

1 Young A, Koduri G, Batley M, et al. Mortality in rheumatoid arthritis. Increased in the early course of disease, in ischaemic heart disease and in pulmonary fibrosis. Rheumatology (Oxford) 2007; 46: 350-357.

2 Bilgici A, Ulusoy $\mathrm{H}$, Kuru $\mathrm{O}$, et al. Pulmonary involvement in rheumatoid arthritis. Rheumatol Int 2005; 25: 429-435.

3 Dawson JK, Fewins HE, Desmond J, et al. Fibrosing alveolitis in patients with rheumatoid arthritis as assessed by high resolution computed tomography, chest radiography, and pulmonary function tests. Thorax 2001; 56: 622-627.

4 Gabbay E, Tarala R, Will R, et al. Interstitial lung disease in recent onset rheumatoid arthritis. Am J Respir Crit Care Med 1997; 156: 528-535.

5 Gochuico BR, Avila NA, Chow CK, et al. Progressive preclinical interstitial lung disease in rheumatoid arthritis. Arch Intern Med 2008; 168: 159-166.

6 Travis WD King TE Jr, Bateman ED, et al. American Thoracic Society/European Respiratory Society international multidisciplinary consensus classification of the idiopathic interstitial pneumonias. Am J Respir Crit Care Med 2002; 165: 277-304.

7 Flaherty KR, Thwaite EL, Kazerooni EA, et al. Radiological versus histological diagnosis in UIP and NSIP: survival implications. Thorax 2003; 58: 143-148.

8 Bjoraker JA, Ryu JH, Edwin MK, et al. Prognostic significance of histopathologic subsets in idiopathic pulmonary fibrosis. Am J Respir Crit Care Med 1998; 157: 199-203.

9 Travis WD, Hunninghake G, King TE Jr, et al. Idiopathic nonspecific interstitial pneumonia: report of an American Thoracic Society project. Am J Respir Crit Care Med 2008; 177: 1338-1347.

10 Bouros D, Wells AU, Nicholson AG, et al. Histopathologic subsets of fibrosing alveolitis in patients with systemic sclerosis and their relationship to outcome. Am J Respir Crit Care Med 2002; 165: 1581-1586.

11 Cottin V, Thivolet-Bejui F, Reynaud-Gaubert M, et al. Interstitial lung disease in amyopathic dermatomyositis, dermatomyositis and polymyositis. Eur Respir J 2003; 22: 245-250.

12 Douglas WW, Tazelaar HD, Hartman TE, et al. Polymyositisdermatomyositis-associated interstitial lung disease. Am J Respir Crit Care Med 2001; 164: 1182-1185.

13 Fujita J, Yoshinouchi T, Ohtsuki Y, et al. Non-specific interstitial pneumonia as pulmonary involvement of systemic sclerosis. Ann Rheum Dis 2001; 60: 281-283.

14 Ito I, Nagai S, Kitaichi M, et al. Pulmonary manifestations of primary Sjögren's syndrome: a clinical, radiologic, and pathologic study. Am J Respir Crit Care Med 2005; 171: 632-638.

15 Kinder BW, Collard HR, Koth L, et al. Idiopathic nonspecific interstitial pneumonia: lung manifestation of undifferentiated connective tissue disease? Am J Respir Crit Care Med 2007; 176: 691-697.

16 Parambil JG, Myers JL, Lindell RM, et al. Interstitial lung disease in primary Sjögren syndrome. Chest 2006; 130: 1489-1495.

17 Tansey D, Wells AU, Colby TV, et al. Variations in histological patterns of interstitial pneumonia between connective tissue disorders and their relationship to prognosis. Histopathology 2004; 44: 585-596.

18 Huh JW, Kim DS, Lee CK, et al. Two distinct clinical types of interstitial lung disease associated with polymyositis-dermatomyositis. Respir Med 2007; 101: 1761-1769.

19 Lee HK, Kim DS, Yoo B, et al. Histopathologic pattern and clinical features of rheumatoid arthritis-associated interstitial lung disease. Chest 2005; 127: 2019-2027.

20 Yoshinouchi T, Ohtsuki Y, Fujita J, et al. Nonspecific interstitial pneumonia pattern as pulmonary involvement of rheumatoid arthritis. Rheumatol Int 2005; 26: 121-125.

21 Hunninghake GW, Zimmerman MB, Schwartz DA, et al. Utility of a lung biopsy for the diagnosis of idiopathic pulmonary fibrosis. Am J Respir Crit Care Med 2001; 164: 193-196.

22 Raghu G, Mageto YN, Lockhart D, et al. The accuracy of the clinical diagnosis of new-onset idiopathic pulmonary fibrosis and other interstitial lung disease: a prospective study. Chest 1999; 116: 1168-1174.

23 Akira $M$, Sakatani $M$, Hara $H$. Thin-section CT findings in rheumatoid arthritis-associated lung disease: CT patterns and their courses. J Comput Assist Tomogr 1999; 23: 941-948.

24 Tanaka N, Kim JS, Newell JD, et al. Rheumatoid arthritis-related lung diseases: CT findings. Radiology 2004; 232: 81-91. 
25 Arnett FC, Edworthy SM, Bloch DA, et al. The American Rheumatism Association 1987 revised criteria for the classification of rheumatoid arthritis. Arthritis Rheum 1988; 31: 315-324.

26 Best AC, Meng J, Lynch AM, et al. Idiopathic pulmonary fibrosis: physiologic tests, quantitative CT indexes, and CT visual scores as predictors of mortality. Radiology 2008; 246: 935-940.

27 Sumikawa H, Johkoh T, Colby TV, et al. Computed tomography findings in pathological usual interstitial pneumonia: relationship to survival. Am J Respir Crit Care Med 2008; 177: 433-439.

28 Yousem SA, Colby TV, Carrington CB. Lung biopsy in rheumatoid arthritis. Am Rev Respir Dis 1985; 131: 770-777.

29 Hakala M, Paakko P, Huhti E, et al. Open lung biopsy of patients with rheumatoid arthritis. Clin Rheumatol 1990; 9: 452-460.

30 Park JH, Kim DS, Park IN, et al. Prognosis of fibrotic interstitial pneumonia: idiopathic versus collagen vascular disease-related subtypes. Am J Respir Crit Care Med 2007; 175: 705-711.
31 Biederer J, Schnabel A, Muhle C, et al. Correlation between HRCT findings, pulmonary function tests and bronchoalveolar lavage cytology in interstitial lung disease associated with rheumatoid arthritis. Eur Radiol 2004; 14: 272-280.

32 Hagiwara K, Sato T, Takagi-Kobayashi S, et al. Acute exacerbation of preexisting interstitial lung disease after administration of etanercept for rheumatoid arthritis. J Rheumatol 2007; 34: 1151-1154.

33 Ostor AJ, Crisp AJ, Somerville MF, et al. Fatal exacerbation of rheumatoid arthritis associated fibrosing alveolitis in patients given infliximab. BMJ 2004; 329: 1266.

34 Villeneuve E, St-Pierre A, Haraoui B. Interstitial pneumonitis associated with infliximab therapy. J Rheumatol 2006; 33: 1189-1193.

35 Wolfe F, Caplan L, Michaud K. Rheumatoid arthritis treatment and the risk of severe interstitial lung disease. Scand J Rheumatol 2007; 36: 172-178. 